Honam Mathematical J. 34 (2012), No. 4, pp. 495-504

http://dx.doi.org/10.5831/HMJ.2012.34.4.495

\title{
SOME REMARKS ON SEMIALGEBRAIC TRANSFORMATION GROUPS
}

\author{
Dae Heui Park
}

\begin{abstract}
Let $G$ be a semialgebraic group and $M$ a proper semialgebraic $G$-set which is locally complete. In this paper we show that the orbit space $M / G$ has a semialgebraic structure such that the orbit map is semialgebraic.
\end{abstract}

\section{Introduction}

A semialgebraic set is a subset of some $\mathbb{R}^{n}$ defined by finite number of polynomial equations and inequalities. Throughout this paper we consider semialgebraic sets in $\mathbb{R}^{n}$ equipped with the subspace topology induced by the usual topology of $\mathbb{R}^{n}$. A continuous map $f: M \rightarrow N$ between semialgebraic sets $M \subset \mathbb{R}^{m}$ and $N \subset \mathbb{R}^{n}$ is called semialgebraic if its graph is a semialgebraic set in $\mathbb{R}^{m} \times \mathbb{R}^{n}$. Note that all semialgebraic maps are assumed to be continuous.

In this paper we discuss topological properties of semialgebraic sets with semialgebraic actions of semialgebraic groups. A semialgebraic set $G$ is called a semialgebraic group if it is a topological group whose multiplication and inversion are semialgebraic. Let $M$ be a semialgebraic set and $G$ a semialgebraic group. We say $M$ is a semialgebraic $G$-set if the action $\theta: G \times M \rightarrow M$ is semialgebraic. A semialgebraic $G$-set $M$ is called proper if it is a (topologically) proper $G$-space. Here is a natural question. Does there exist a semialgebraic structure of the orbit space $M / G$ such that the orbit map is semialgebraic? We propose a partially positive answer of this question as follows.

Received July 27, 2012. Accepted September 10, 2012.

2010 Mathematics Subject Classification. 57Sxx, 14P10, 54B15, 57S20.

Key words and phrases. transformation groups, semialgebraic sets, orbit spaces.

This study was financially supported by Chonnam National University, 2008 
Theorem 1.1. Let $G$ be a semialgebraic group and $M$ a proper semialgebraic $G$-set which is locally complete. Then $M / G$ has a semialgebraic structure such that the orbit map $\pi: M \rightarrow M / G$ is semialgebraic.

This theorem was proved by Brumfiel if $G$ is compact in [3].

This paper is organized as follows. In Section 2 we review some background materials on proper $G$-spaces. In Section 3 we prove Theorems 1.1. It also contains some properties related to proper semialgebraic $G$-sets. In Section 4 we give a construction of a section-like subset of a proper semialgebraic $G$-set.

\section{Proper $G$-spaces}

We first give the definition and some basic properties of proper actions. For the details, we refer the reader to [6] and [5].

Definition 2.1. Let $G$ be a topological group. A Hausdorff $G$-space $X$ is called by Palais [6] a proper $G$-space if each $x \in X$ has a neighborhood $U$ satisfying that for any $y \in X$ there exists a neighborhood $V$ of $y$ such that the closure of the set

$$
((U, V))=\{g \in G \mid g U \cap V \neq \emptyset\}
$$

is compact.

From now on, $G$ denotes a Lie group. The followings are basic results about proper actions.

Proposition $2.2([6])$. Let $X$ be a proper $G$-space which is completely regular, and let $x \in X$. Then,

(1) the orbit $G(x)$ is closed in $X$,

(2) the isotropy group $G_{x}$ is compact,

(3) the evaluation map $\theta_{x}: G \rightarrow G(x), \theta_{x}(g)=g x$ is open,

(4) the map $f: G / G_{x} \rightarrow G(x), f\left(g G_{x}\right)=g x$ is a homeomorphism,

(5) the orbit space $X / G$ is completely regular,

(6) if $H$ is a closed subgroup of $G$ and $Y$ is an $H$-invariant subspace of $X$ then $Y$ is a proper $H$-space.

Definition 2.3. Let $X$ be a $G$-space and $H$ a closed subgroup of $G$. A subset $S$ of $X$ is called an $H$-kernel if there exists a continuous $G$-map $f: G S \rightarrow G / H$ such that $f^{-1}(e H)=S$, where $e$ is the identity of $G$. If, in addition, $G S$ is open in $X$, then $S$ is called $H$-slice in $X$. For $x \in X$ a slice at $x$ means a $G_{x}$-slice $S$ in $X$ such that $x \in S$. 
When $G$ is compact, it is well-known that a subset $S$ of $X$ is an $H$ kernel if and only if $G S$ is $G$-homeomorphic to $G \times_{H} S$ (see [2]). We now show that the same is true for any locally compact topological group $G$ if $H$ is compact and $X$ is first-countable. Note that every Lie group is locally compact.

Proposition 2.4. Let $X$ be a $G$-space and $H$ a compact subgroup of $G$. Let $S \subset X$ be an H-kernel. Then the action $\theta: G \times S \rightarrow G S$ is proper.

Proof. Since we do not need to mention about $X$, we assume $X=G S$. Let $f: X \rightarrow G / H$ be a continuous $G$-map such that $f^{-1}(e H)=S$. Clearly $S$ is closed in $X$. Let $\pi: G \rightarrow G / H$ be the quotient map. Since $H$ is compact, $\pi$ is a proper map. For a compact subset $C$ of $X$, its image $f(C)$ is compact in $G / H$, hence $B=\pi^{-1}(f(C))$ is a compact subset of $G$. Let $B^{-1}=\left\{g^{-1} \in G \mid g \in B\right\}$. Then $\theta^{-1}(C)$ is a closed subset of a compact subset $B \times \theta\left(B^{-1} \times C\right)$ of $G \times S$, hence $\theta^{-1}(C)$ is compact. This shows that $\theta$ is proper.

We know that a proper map between two first-countable spaces is closed.

Corollary 2.5. Let $X$ be a first-countable $G$-spaces. Let $H$ be a compact subgroup of $G$. If $S$ is an $H$-kernel of $X$, then $G S$ is $G$ homeomorphic to $G \times_{H} S$. Moreover, $S / H$ is homeomorphic to $G S / G$.

Proof. It is elementary to show that the action $\theta: G \times S \rightarrow G S$ is reduced to a bijective continuous map $\bar{\theta}: G \times_{H} S \rightarrow G S$. The action $\theta$ is proper by Proposition 2.4. Besides, since $X$ is first-countable, it follows that $\theta$ is closed. So $\bar{\theta}$ is a closed map, and hence a homeomorphism.

We now show that $S / H$ is homeomorphic to $G S / G$. The inclusion map $i: S \hookrightarrow G S$ obviously induces a continuous map $\bar{i}: S / H \rightarrow G S / G$, so we only need to construct the inverse map. Now the second projection $p: G \times S \rightarrow S$ induces a continuous map $\bar{p}: G \times{ }_{H} S \rightarrow S / H$ and we get a continuous map $f=\bar{p} \circ \bar{\theta}^{-1}: G S \rightarrow S / H$ which induces the desired continuous map $\bar{f}: G S / G \rightarrow S / H$ so that $\bar{f}=\bar{i}^{-1}$.

The following proposition is one of the main results of [6].

Proposition 2.6. Let $G$ be a Lie group and $X$ a proper $G$-space. Then for each $x \in X$ there exists a slice at $x$. 


\section{3. proper semialgebraic $G$-sets}

In this section we prove Theorems 1.1. We first gather some properties concerning semialgebraic sets and maps without proofs, which will be used in this paper. For the details, we refer the reader to [1] and [4].

The class of semialgebraic sets in $\mathbb{R}^{n}$ is the smallest collection of subsets containing all subsets of the form $\left\{x \in \mathbb{R}^{n} \mid p(x)>0\right\}$ for a real valued polynomial $p(x)=p\left(x_{1}, \ldots, x_{n}\right)$, which is stable under finite union, finite intersection and complement. Since every semialgebraic set is a metric space, it is first-countable and completely regular. Recall that a continuous map $f: X \rightarrow Y$ between semialgebraic sets $X\left(\subset \mathbb{R}^{m}\right)$ and $Y\left(\subset \mathbb{R}^{n}\right)$ is called semialgebraic if its graph is a semialgebraic set in $\mathbb{R}^{m} \times \mathbb{R}^{n}$.

Proposition 3.1. (1) Every semialgebraic set has a finite number of connected components which are also semialgebraic.

(2) Composition of two semialgebraic maps is semialgebraic.

(3) Let $f: X \rightarrow Y$ be a semialgebraic map. If $A \subset X$ is semialgebraic, then its image $f(A)$ is semialgebraic. If $B \subset Y$ is semialgebraic, then its inverse image $f^{-1}(B)$ is semialgebraic.

(4) If $A \subset X$ is semialgebraic, then the closure $\bar{A}$, the interior $\stackrel{\circ}{A}$ and the complement $A^{c}$ in $X$ are all semialgebraic.

(5) Let $f: X \rightarrow Q$ and $g: X \rightarrow Y$ be semialgebraic. Assume $f$ is surjective. If $h: Q \rightarrow Y$ is a continuous map such that $h \circ f=g$, then $h$ is semialgebraic.

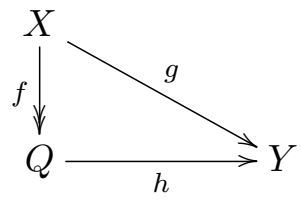

(6) If $f: X \rightarrow Y$ is a semialgebraic map which is a homeomorphism, then the inverse $f^{-1}$ is also semialgebraic.

The definition of a semialgebraic group is given obviously, i.e., a semialgebraic set $G \subset \mathbb{R}^{n}$ is called a semialgebraic group if it is a topological group such that the group multiplication and the inversion are semialgebraic. If $H$ is a subgroup and semialgebraic subset of a semialgebraic group $G$, then $H$ is called a semialgebraic subgroup of $G$.

Proposition 3.2. (1) Every semialgebraic group has a Lie group structure, and hence locally compact. 
(2) Every semialgebraic subgroup of a semialgebraic group is closed.

Proof. The results of this proposition were proved in the o-minimal structures(see, $[7,8])$. Since semialgebraic structure is a special case of $o$-minimal structure, the proof is complete.

Let $M$ be a semialgebraic $G$-set. If $G$ is compact then $M$ is a proper semialgebraic $G$-set. If $G$ is noncompact but $M$ is compact then $M$ is not proper.

For topological proper actions the following proposition appears in [5, Section 1.3] whose proofs are straightforward.

Proposition 3.3. Let $M$ be a proper semialgebraic $G$-set and let $x \in M$, then

(1) the isotropy subgroup $G_{x}$ is compact and semialgebraic,

(2) the orbit $G(x)$ is a closed semialgebraic subset of $M$,

(3) the fixed point set $M^{G}=\{x \in M \mid g x=x$ for all $g \in G\}$ is a closed semialgebraic subset of $M$.

We now treat a semialgebraic structure of the orbit space of a proper semialgebraic $G$-set.

Definition 3.4. Let $M$ be a semialgebraic set and $E \subset M \times M$ an equivalence relation in $M$. Assume $E$ is a closed semialgebraic subset of $M \times M$. Let $p_{1}, p_{2}: E \rightarrow M$ be the restrictions of the canonical projections $M \times M \rightarrow M$ to each factors. We call a closed semialgebraic subset $Y$ of $M$ is a section-like subset of $M$ for $E$ if the map $\left.p_{1}\right|_{p_{2}^{-1}(Y)}: p_{2}^{-1}(Y) \rightarrow M$ is proper and surjective.

Let $M$ be a proper semialgebraic $G$-set. Then the associated action

$$
\vartheta_{*}: G \times M \rightarrow M \times M, \quad(g, x) \mapsto(g x, x)
$$

is proper, and hence closed. Let $E_{G}$ be the semialgebraic equivalence relation corresponding to the action, that is, $E_{G}=\{(g x, x) \in M \times M \mid$ $g \in G, x \in M\}$. Since $\vartheta_{*}$ is semialgebraic and closed, $E_{G}=\vartheta_{*}(G \times M)$ is a closed semialgebraic subset of $M \times M$. For each open semialgebraic subset $U$ of $M$, the set $p_{1}\left(p_{2}^{-1}(U)\right)=G U$ is open in $M$. Thus $E_{G}$ is open over $M$ (cf. [10, Lemma 6.1]). Therefore $E_{G}$ is a closed semialgebraic equivalence relation on $M$ which is open over $M$.

The following proposition tell us when a semialgebraic structure of the orbit space exists.

Proposition 3.5. Let $M$ be a semialgebraic $G$-set. Then the following are equivalent. 
(1) $M / G$ has a semialgebraic structure such that the orbit map $\pi: M \rightarrow$ $M / G$ is semialgebraic.

(2) There exists a section-like subset of $M$ for $E_{G}$.

If these conditions are satisfied, the inclusion $Y \hookrightarrow M$ induces a semialgebraic homeomorphism $Y / E_{Y} \rightarrow M / G=M / E_{G}$ where $E_{Y}=$ $E_{G} \cap(Y \times Y)$.

Proof. See Theorem 5.1 and Corollary 7.3 of [10].

We now prove Theorem 1.1.

Proof of Theorem 1.1. By Theorem 6.2 of [10], $M / G$ has a semialgebraic structure $S$ (which is not necessarily affine) such that the orbit map $\pi^{\prime}: M \rightarrow S$ is semialgebraic and continuous. By Proposition 2.2(5) the orbit space $M / G$ is regular, so is $S$. By Theorem 1 of [9] there exist a semialgebraic set $Z \subset \mathbb{R}^{k}$ and a semialgebraic homeomorphism $\phi: S \rightarrow Z$. Therefore $Z$ is a desired semialgebraic structure of $M / G$.

From Theorem 1.1 and Proposition 3.5 we have the following corollary.

Corollary 3.6. Let $M$ be a proper semialgebraic $G$-set which is locally complete. Then there is a section-like subset $Y$ of $M$.

In Section 4 we construct a section-like subset of $M$.

\section{Some remarks}

If $M$ is a proper semialgebraic $G$-set which is locally complete, then there exists a section-like subset $Y$ of $M$ (Corollary 3.6). In this section we construct $Y$. Before we do this, let us investigate some of the properties related with section-like sets.

Proposition 4.1. Let $Y$ be a section-like subset of a proper semialgebraic $G$-set $M$, then the action $\theta: G \times Y \rightarrow M$ is proper.

Proof. Note that

$$
\begin{aligned}
p_{2}^{-1}(Y) & =\left\{(x, y) \in E_{G} \mid y \in Y\right\} \\
& =\{(g y, y) \in M \times M \mid g \in G, y \in Y\} .
\end{aligned}
$$

Let $h$ denote the restriction $\left.p_{1}\right|_{p_{2}^{-1}(Y)}: p_{2}^{-1}(Y) \rightarrow M$ of $p_{1}$ to $p_{2}^{-1}(Y)$. Then $h\left(p_{2}^{-1}(Y)\right)=\cup_{y \in Y} G(y)$. 
To show that $\theta$ is proper, let $C$ be a compact subset of $M$. Since $h$ is proper, the set $A:=p_{2}\left(h^{-1}(C)\right)$ is a compact subset of $Y$. Note that $h^{-1}(C)=\{(g y, y) \in M \times M \mid y \in Y, g y \in C, g \in G\}$, hence we have

$$
A=\{y \in Y \mid g y \in C \text { for some } g \in G\} \text {. }
$$

On the other hand

$$
\theta^{-1}(C)=\{(g, y) \in G \times Y \mid g y \in C\} .
$$

Thus $\theta^{-1}(C)$ is a subset of $((A, C)) \times A$. Since $\theta^{-1}(C)$ is a closed subset of compact set $((A \cup C, A \cup C)) \times A$, it is compact, and hence $\theta$ is proper.

Note that a semialgebraic set is locally complete if and only if it is locally compact.

Proposition 4.2. Let $M$ be a proper semialgebraic $G$-set which is locally complete, and let $Y$ be a section-like subset of $M$. Then the restriction $\left.\pi\right|_{Y}: Y \rightarrow M / G$ of the orbit map is proper.

Proof. First, we show that $\left.\pi\right|_{Y} ^{-1}(z)$ is compact for all $z \in M / G$. For $z \in M / G$, let $x$ be a point of $\pi^{-1}(z)$. Then we have

$$
\left.\pi\right|_{Y} ^{-1}(z)=Y \cap \pi^{-1}(z)=Y \cap G(x)=p_{2}\left(h^{-1}(x)\right) .
$$

Hence $\left.\pi\right|_{Y} ^{-1}(z)$ is compact.

Next, to prove that $\left.\pi\right|_{Y}$ is closed by showing that if $\left\{y_{n}\right\}$ is a sequence in $Y$ such that $\left\{\pi\left(y_{n}\right)\right\}$ converges to some $z \in M / G$, then there exists a convergent subsequence of $y_{n}$ which converges to an element in $Y$. To show this, let $\left\{y_{n}\right\}$ be a sequence in $Y$ such that $\left\{\pi\left(y_{n}\right)\right\}$ converges to $z \in M / G$. Choose $x$ in $\left.\pi\right|_{Y} ^{-1}(z)$. Since $M$ is locally complete, we can take a compact neighborhood $U$ of $x$ in $M$. For simplicity we may assume that $\pi\left(y_{n}\right) \in \pi(U)$ for all $n$. Since $p_{2}\left(h^{-1}(U)\right)$ is compact and $y_{n} \in$ $p_{2}\left(h^{-1}(U)\right)$ for all $n$, the sequence $\left\{y_{n}\right\}$ has a convergent subsequence which converges to an element in $Y$.

Proposition 4.3. Let $M$ be a semialgebraic $G$-set and $H$ a compact semialgebraic subgroup of $G$. Let $S \subset M$ be a semialgebraic $H$-kernel. Then $S$ is a section-like subset of the semialgebraic $G$-set $G S$. Moreover $S / H$ is semialgebraically homeomorphic to $G S / G$.

Proof. First we show that $h=\left.p_{1}\right|_{p_{2}^{-1}(S)}: p_{2}^{-1}(S) \rightarrow G S$ is proper and surjective. Since $p_{2}^{-1}(S)=\{(g y, y) \mid g \in G, y \in S\}$ and $h(g y, y)=g y$, the map $h$ is surjective. For a compact subset $C$ of $G S$, its inverse image $h^{-1}(C)=\left\{(g y, y) \in p_{2}^{-1}(S) \mid g y \in C\right\}$ is a closed subset of $C \times D$ where 
$D=\{y \in M \mid g y \in C\}=\pi_{2}\left(\theta^{-1}(C)\right), \theta: G \times S \rightarrow G S$ is the action, and $\pi_{2}: G \times S \rightarrow S$ is the projection to the second factor. Since $\theta$ is proper by Proposition 2.4, $D$ is compact, and hence $h^{-1}(C)$ is compact.

The last statement follows from Corollary 2.5 since the homeomorphism $S / H \rightarrow G S / G$ is obviously semialgebraic.

We now give a specific construction of a section-like subset of a proper semialgebraic $G$-set.

Theorem 4.4. Let $M$ be a proper semialgebraic $G$-set which is locally complete. Then there exists a section-like subset $Y$ of $M$ such that $Y=\bar{S}$ where $S$ is the image of a (noncontinuous) semialgebraic cross section of the orbit map $\pi: M \rightarrow M / G$.

Proof. We may assume that $M$ is a closed subset of $\mathbb{R}^{n}$. Indeed, let $M$ be a semialgebraic set which is not closed in $\mathbb{R}^{n}$. Since $M$ is locally complete, the set $U=\left(\mathbb{R}^{n}-\bar{M}\right) \cup M$ is an open semialgebraic subset of $\mathbb{R}^{n}$ where $\bar{M}$ is the closure of $M$ in $\mathbb{R}^{n}$. For each $x \in \mathbb{R}^{n}$, we define the distance from $x$ to $\mathbb{R}^{n}-U$ by the equation

$$
\left.\operatorname{dist}\left(x, \mathbb{R}^{n}-U\right)=\inf \left\{\|x-y\| \mid y \in \mathbb{R}^{n}-U\right)\right\} .
$$

Then the map

$$
f: M \rightarrow \mathbb{R}^{n+1}, f(x)=\left(x, \frac{1}{\operatorname{dist}\left(x, \mathbb{R}^{n}-U\right)}\right)
$$

is a semialgebraic embedding such that $f(M)$ is a closed semialgebraic subset of $\mathbb{R}^{n+1}$.

Since $M$ is a proper $G$-space, for each $x \in M$, the orbit $G(x)$ is closed in $M$, and hence it is closed in $\mathbb{R}^{n}$. From each orbit $G(x)$ choose a point $s(x)$ which is closest to the origin $\mathbf{0}$ of $\mathbb{R}^{n}$. Such $s(x)$ exists because $G(x)$ is closed in $\mathbb{R}^{n}$. If there are more than one such points, choose a point whose coordinate is the smallest with respect to the lexicographical order of the coordinates of $\mathbb{R}^{n}$. Then the set-theoretic map $s: M \rightarrow M$, $x \mapsto s(x)$ may not be continuous but its graph is semialgebraic. Indeed, first consider a semialgebraic set

$$
Z_{0}=\left\{(x, y) \in \mathbb{R}^{2 n} \mid \exists g \in G y=g x \text { and } \forall g^{\prime} \in G\|y\|^{2} \leq\left\|g^{\prime} x\right\|^{2}\right\} .
$$

Next, from $Z_{0}$, we obtain a new semialgebraic set

$$
Z_{1}=\left\{\left(x, y_{1}, \ldots, y_{n}\right) \in Z_{0} \mid \forall\left(x, z_{1}, \ldots, z_{n}\right) \in Z_{0} y_{1} \leq z_{1}\right\} .
$$

Continue the process, we obtain semialgebraic sets

$$
Z_{i+1}=\left\{\left(x, y_{1}, \ldots, y_{n}\right) \in Z_{i} \mid \forall\left(x, z_{1}, \ldots, z_{n}\right) \in Z_{i} y_{i+1} \leq z_{i+1}\right\}
$$


for $i=0, \ldots, n-1$. Then the semialgebraic set $Z_{n}$ is the graph of $s$, so it follows that the image $S=\{s(y) \mid y \in M\}$ is a semialgebraic subset of $M$.

Let $Y$ denote the closure of $S$ in $M$. Then $Y$ is a closed semialgebraic subset of $M$. We claim that $Y$ is section-like. Let $h=$ $\left.p_{1}\right|_{p_{2}^{-1}(Y)}: p_{2}^{-1}(Y) \rightarrow M$. The subjectivity of $\left.p_{1}\right|_{p_{2}^{-1}(Y)}$ is clear. We now prove $h$ is proper. Let $C$ be a compact subset of $M$. Since $h$ is continuous, $h^{-1}(C)$ is closed. To show that $h^{-1}(C)$ is bounded it is enough to show that both $p_{1}\left(h^{-1}(C)\right)$ and $p_{2}\left(h^{-1}(C)\right)$ are bounded. Clearly $p_{1}\left(h^{-1}(C)\right)=C$ is bounded. On the other hand, there is a positive real number $r$ such that $\|x\|<r$ for all $x \in C$. For $x \in C$, $p_{2}\left(h^{-1}(x)\right)=G(x) \cap Y$. Let $y \in p_{2}\left(h^{-1}(x)\right)$, then $y=g x$ for some $g \in G$. Since $Y=\bar{S}$, there is a sequence $\left\{z_{n}\right\}$ in $S$ which converges to $y$. Then $\left\{g^{-1} z_{n}\right\}$ converges to $x$. From the definition of the set $S$, $\left\|z_{n}\right\| \leqq\left\|g^{-1} z_{n}\right\|<\|x\|+1$ for sufficiently large $n$. Since $\left\{z_{n}\right\}$ converges to $y,\|y\| \leqq\|x\|+1<r+1$. Thus $p_{2}\left(h^{-1}(x)\right)$ is bounded, so it follows that $p_{2}\left(h^{-1}(C)\right)$ is bounded.

According to Proposition 3.5, Theorem 4.4 gives another proof of Theorem 1.1.

From Propositions 3.5 and 4.2 and Theorem 4.4 we have the following theorem.

Theorem 4.5. Let $M$ be a proper semialgebraic $G$-set which is locally complete. Then we obtain the following:

(1) There exists a closed, semialgebraic, section-like subset $Y$ of $M$ for $E_{G}$, and thus $Y / E_{Y}$ has a semialgebraic structure such that the restriction of the quotient map $Y \rightarrow Y / E_{Y}$ is semialgebraic and proper, where $E_{Y}=E_{G} \cap(Y \times Y)$.

(2) Hence $M / G$ has a semialgebraic structure such that the orbit map $\pi: M \rightarrow M / G$ is semialgebraic. Moreover, the inclusion $Y \hookrightarrow$ $M$ induces a semialgebraic homeomorphism $Y / E_{Y} \rightarrow M / G(=$ $\left.M / E_{G}\right)$.

If $G$ is compact, we can take $Y=M$, thus it is clear that $M / G$ has a semialgebraic structure, which is one of the main results of [3].

\section{References}

[1] J. Bochnak, M. Coste and M.-F. Roy, Real Agebraic Geometry, Erg. der Math. und ihrer Grenzg., vol. 36, Springer-Verlag, Berlin Heidelberg, 1998. 
[2] G. E. Bredon, Introduction to Compact Transformation Groups, Pure and Applied Mathematics, vol. 46, Academic Press, New York, 1972.

[3] G. W. Brumfiel, Quotient space for semialgebraic equivalence relation, Math. Z. 195 (1987), 69-78.

[4] H. Delfs and M. Knebusch, Locally Semialgebraic Spaces, Lecture Notes in Math. 1173, Springer, Berlin, 1985.

[5] T. tom Dieck, Transformation Groups, Walter de Gruyter, New York, 1987.

[6] R. S. Palais, On the existence of slices for actions of non-compact Lie groups, Ann. of Math. 73(2) (1961), 295-323.

[7] Y. Peterzil, A. Pillay, and S. Starchenko, Definably simple groups in o-minimal structures, Trans. Amer. Math. Soc. 352(10) (2002), 4397-4419.

[8] A. Pillay, On groups and fields definable in o-minimal structures, J. Pure Applied Algebra 53 (1988), 239-255.

[9] R. Robson, Embedding semi-algebraic spaces, Math. Z. 183 (1983), 365-370.

[10] C. Scheiderer, Quotients of semi-algebraic spaces, Math. Z. 201 (1989), 249-271.

Dae Heui Park

Department of Mathematics, Chonnam National University, Gwangju 500-757, Korea.

E-mail: dhpark3331@jnu.ac.kr 